\title{
Prise en charge des troubles d'articulé phonatoire chez l'enfant
}

\section{RÉSUMÉ}

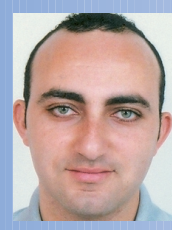

Hicham BENYAHIA*

Spécialiste en ODF,

CES B Orthopédie dento-faciale (2005),

Université Victor Segalen Bordeaux2,

Faculté de médecine dentaire,

BP 6212, Instituts Bab Al Irfane,

Rabat - Maroc.

Loubna BAHIJE

Spécialiste en ODF.

Service d'Orthopédie Dento-Faciale,

Faculté de médecine dentaire,

BP 6212, Instituts Bab Al Irfane,

Rabat - Maroc

\section{Fatima ZAOUI}

Professeur d'enseignement supérieur,

Service d'Orthopédie Dento-Faciale,

Faculté de médecine dentaire,

BP 6212, Instituts Bab Al IIfane,

Rabat - Maroc

\section{Elhoussine AALLOULA}

Professeur d'enseignement supérieur,

Chef de servide d'orthopédie dento-faciale, Service d'Orthopédie Dento-Faciale,

Faculté de médecine dentaire,

BP 6212, Instituts Bab Al Irfane,

Rabat - Maroc.
* Pour toute demande de tiré à part s'adresser à l'auteur à l'adresse :

Service d'Orthopédie Dento-Faciale,

Faculté de médecine dentaire,

BP 6212, Institut Bab Al Irfane

Rabat - Maroc
Les fonctions de la sphère oro-faciale, notamment la ventilation et la déglutition jouent un rôle important dans la morphogenèse des arcades dentaires.

Tous les auteurs ne sont pas unanimes en ce qui concerne la phonation, qui a suscité beaucoup de controverses quant à sa contribution morphogénétique.

Les travaux récents, tendent à replacer cette fonction majeure à sa vraie valeur au sein de la sphère oro-faciale.

Les troubles d'articulé phonatoire intéressent de plus en plus l'orthodontiste, puisqu'ils peuvent être à l'origine de malocclusions dento-alvéolaires.

L'examen fonctionnel permet de préciser ces appuis linguaux défectueux lors de l'articulation des phonèmes, pour orienter une rééducation phonétique réfléchie et bien ciblée. 


\section{Introduction}

$>$

Les fonctions de la sphère oro-faciale,

notamment la ventilation et la déglutition, jouent un rôle important dans la morphogenèse des arcades dentaires. La phonation n'est pas en reste quant à cette contribution morphogénétique. Il est primordial de tenir compte des troubles de l'articulé phonatoire, lors de l'examen clinique fonctionnel, qui sont

\section{Historique}

Cette fonction avait déjà suscité l'intérêt au 19 siècle ; Alexandre Dumas père (1867) cité par Meng[21] était précurseur en affirmant que certains troubles de l'occlusion chez les anglais s'expliquaient par la phonétique particulière notamment du «th» anglo-saxon. II écrivait «le corps mou (langue) l'a emporté sur le corps dur (dents)». II annonçait les travaux

\section{Phonation}

\section{Physiologie}

La phonation est une fonction à but de communication. Elle fait appel à un ensemble de mécanismes physiologiques complexes qui intéressent successivement, les poumons, le pharyngo-larynx, les cavités buccale et nasale, effecteurs terminaux de l'articulation phonétique, où le son parlé trouve sa personnalité sous l'influence directrice du système nerveux[1, 8, 22].

Les modulateurs tels les lèvres, la langue et le voile du palais vont transformer les sons en souvent associés à une déglutition atypique. Leur rééducation est indiquée notamment en présence de malocclusions dento-alvéolaires. Elle nécessite tout de même, pour la réussite et la pérennité des résultats, une bonne maturité intellectuelle et une motivation de l'enfant et de son entourage.

d'un certain nombre d'auteurs (Cauhépé 1953, Fieux 1961 et Bouvet 1965)[5, 6, 12] qui ont étudié toutes les interrelations de la langue avec les arcades dentaires. Et c'est à Husson (1962)[16] que revient le mérite d'avoir démontré que les praxies du langage mettent en œuvre des stéréotypes empruntés aux praxies de la déglutition[3, 16].

phonèmes afin de constituer les mots. La langue, organe le plus important du langage joue un rôle essentiel dans la production des timbres vocaliques, elle détermine le point d'articulation[1, 16, 22].

\section{Évolution}

L'enfant acquiert progressivement les différents phonèmes utiles dans sa langue maternelle. L'âge d'acquisition est très variable d'un enfant à l'autre, mais en moyenne les voyelles sont acquises à 3 ans, les consonnes occlusives 
à 4 ans, et à 7 ans en moyenne, l'enfant peut prononcer n'importe quel phonème[14].

\section{Articulation des phonèmes}

La parole est composée d'unités phonétiques juxtaposées: les phonèmes. Le phonème (terme d'origine grecque) = son de voix, est la plus petite unité phonique perceptible[22].

Lorsque l'on s'exprime, les voyelles sont émises arcades séparées, sans appui lingual. Les consonnes sont émises langue en appui sur le palais, cet appui étant différent selon la consonne. En français aucun phonème ne se prononce langue en appui sur les arcades dentaires contrairement aux langues anglaise, espagnole ou portugaise[11].

Si les appuis linguaux viennent à se modifier pour s'effectuer au niveau dentaire, une malocclusion peut se développer et ce, d'autant plus que la musculature périphérique est défaillante. De ce fait, pour les orthodontistes, le contrôle des appuis musculaires est plus important que le contrôle de la qualité du langage oral.
Les palatogrammes ont donc vu le jour à partir de ce constat : il s'agit d'une méthode simple permettant l'étude des appuis linguaux, en badigeonnant la langue avec un mélange colorant. Le sujet referme la bouche et prononce le phonème convenu, ce qui permettra d'étudier après photographie occlusale aussi bien la forme que l'étendue des lieux d'articulation de la langue pendant la phonation $[3,4]$ (fig. 1).

\section{> Consonnes linguo-palatales antérieures $(T, D, N)$}

Elles présentent l'énorme intérêt d'avoir des sites appositionnels identiques à ceux de la déglutition $[4,5]$ : le lieu d'articulation au niveau de la papille rétro-incisive[1, 5] (fig. 2 a et b).

Anormalement, la pointe de la langue touche les incisives supérieures ou bien s'interpose entre les dents, ou même appuie derrière les incisives inférieures[13] (fig. 3 a-c).

Souvent, pendant la déglutition, la langue n'est pas visible. Une position erronée de la langue ne sera décelable que lors de

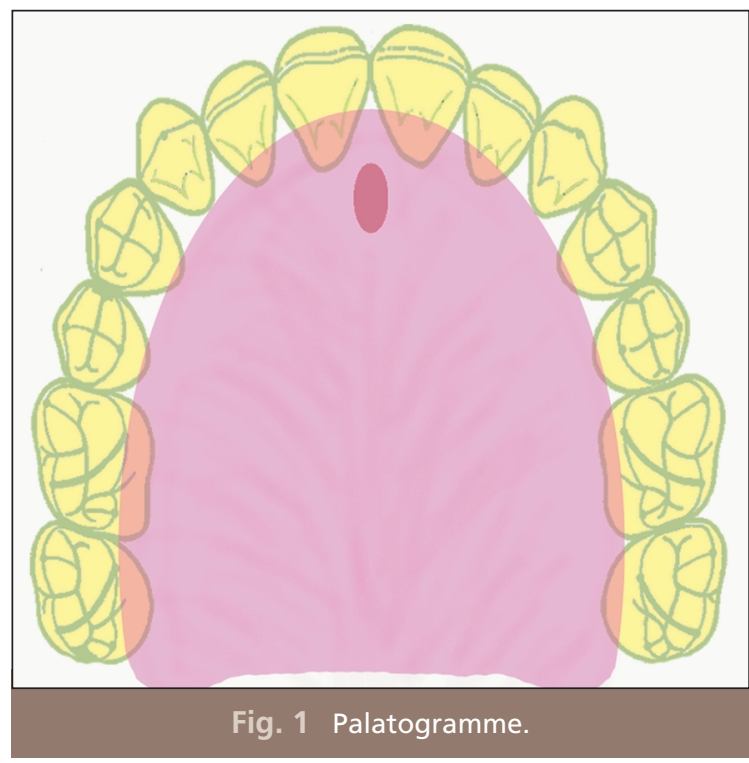



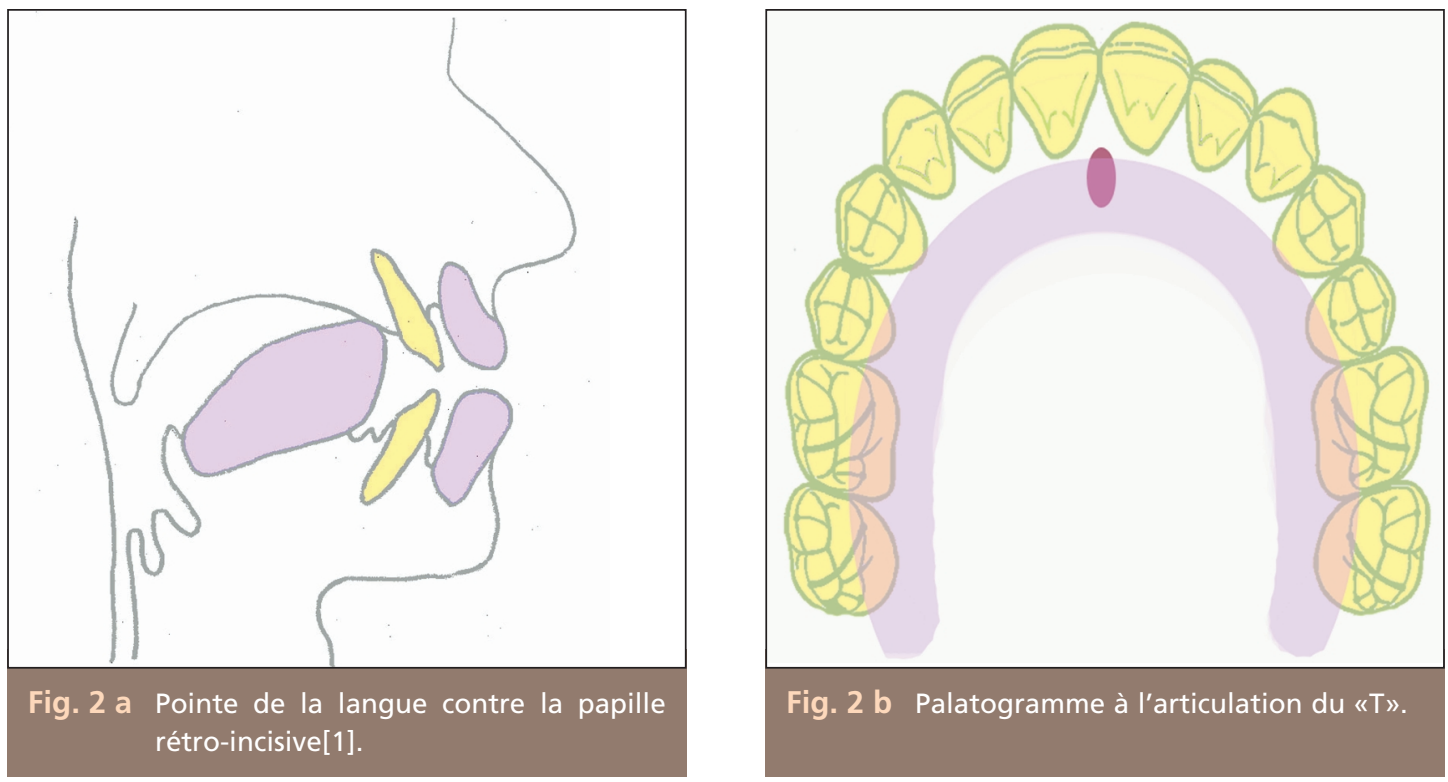

Fig. 2 b Palatogramme à l'articulation du «T».
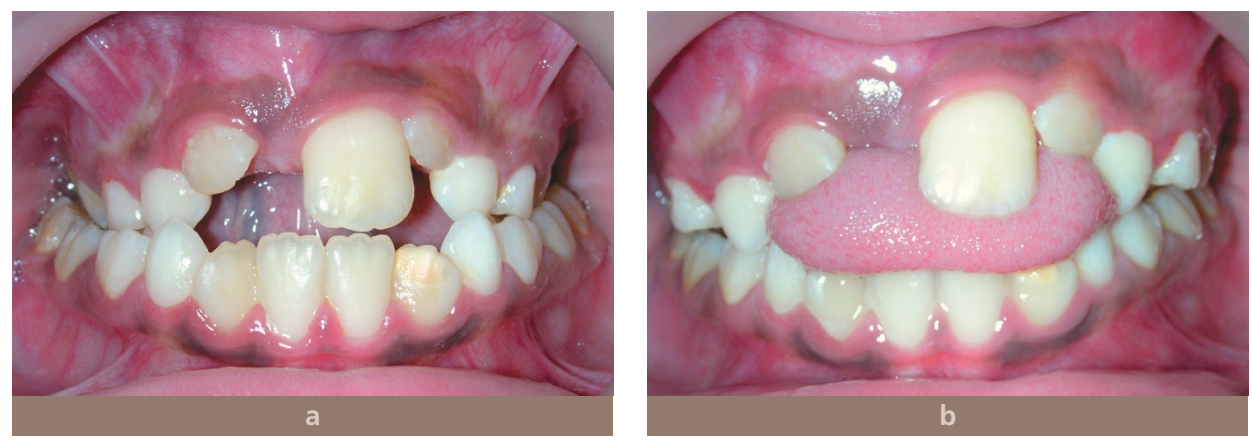

Fig. 3 a et b Béance antérieure avec inclusion de la 11 ; déglutition atypique.

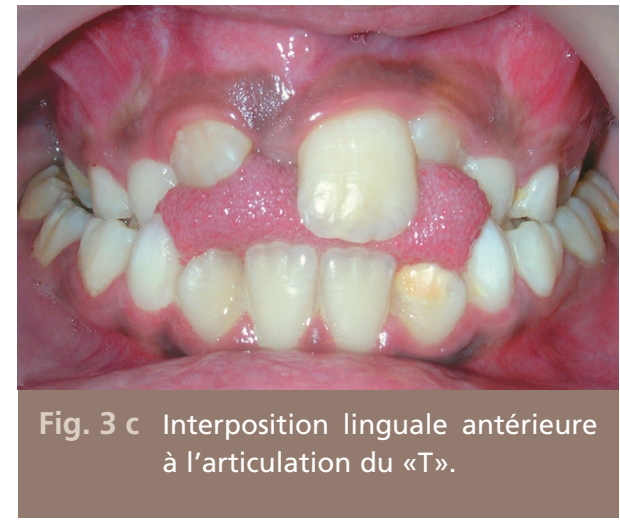

I'articulation des alvéolo-dentales $\mathrm{D}, \mathrm{N}$ et $\mathrm{T}$; donc un rôle diagnostique certain de la posture linguale et de la fonction de déglutition[13].

\section{> Consonnes linguo-palatales moyennes ( $L$ )}

La pointe de la langue se situe derrière la papille rétro-incisive (linguo-palatal moyen) (fig. 4 a et b). L'appui peut devenir apico-dental interférant avec les incisives supérieures en présence de déglutition primaire[1]. Une mauvaise position de la pointe de la langue à la prononciation du phonème $L$ est assez rare.
Elle ne se voit que dans des cas graves d'immaturité motrice de la langue, fatigue ou stress excessif[13].

\section{> Consonnes linguo-palatales latérales $(\mathrm{Ch}, \mathrm{J})$}

C'est une consonne constrictive médiane: la zone de striction se trouve située entre le dos de la langue et le palais dur (fig. 5). Anormalement la langue appuie sur les incisives ou s'interpose entre les dents, antérieurement, ou latéralement à l'origine d'une infra-alvéolie molaire et d'une supraclusion incisive[4] (fig. 6 $a$ et $b)$. 

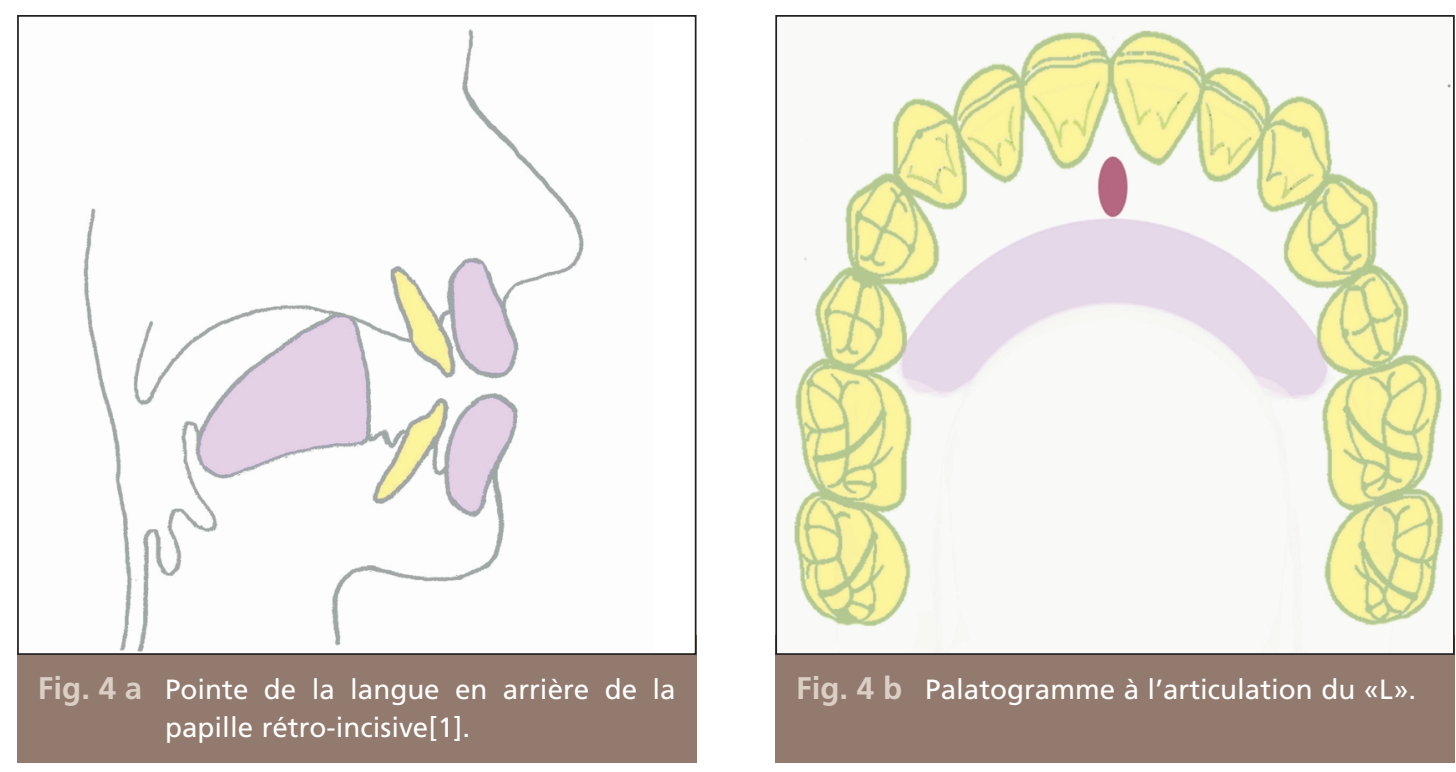

Fig. 4 b Palatogramme à l'articulation du «L».

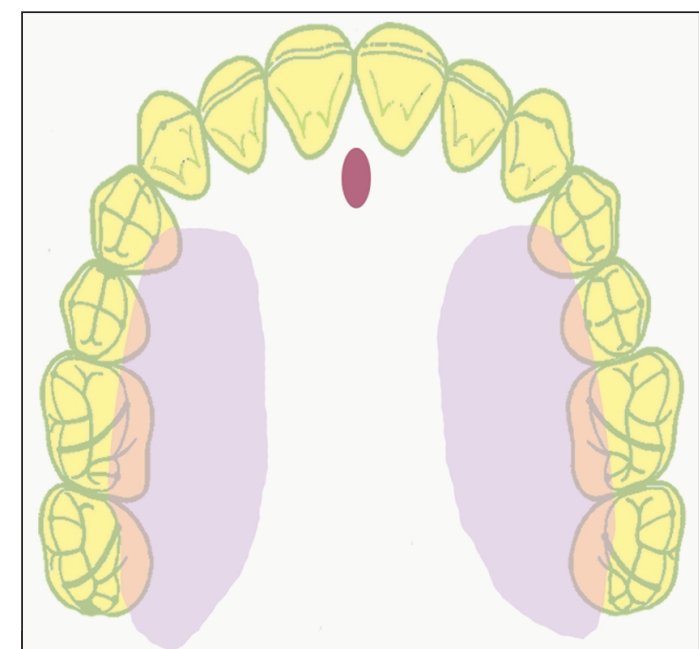

Fig. 5 Palatogramme à l'articulation du «Ch».
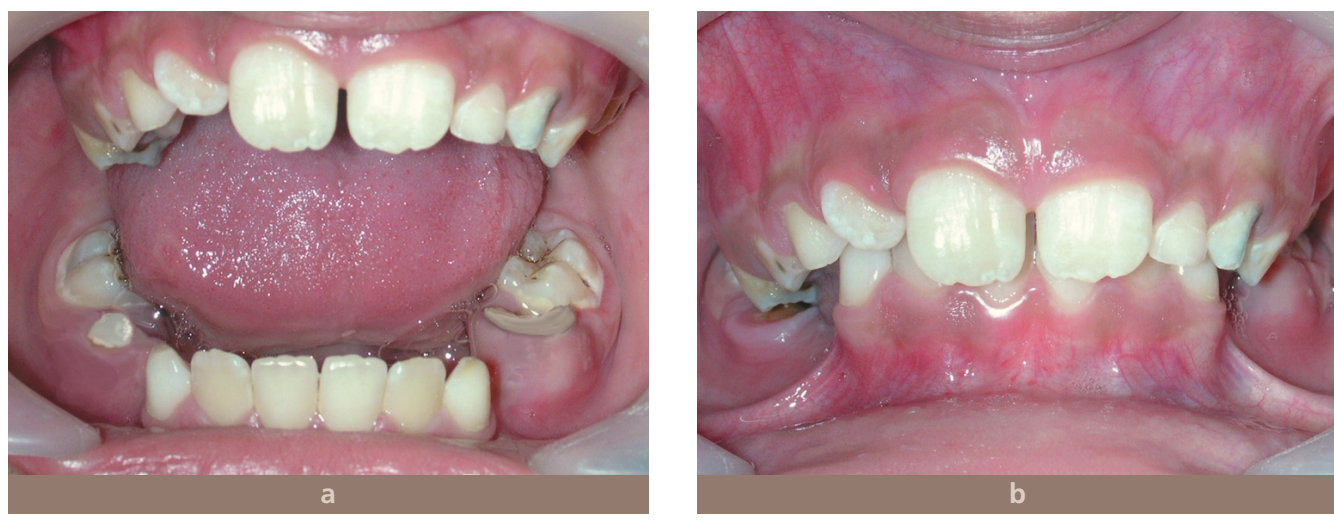

Fig. 6 a et b Étalement lingual latéral entraînant une infraclusie molaire et une supraclusie incisive. 


\section{> Consonnes linguo-dentales (S, Z)}

Consonnes constrictives, la pointe de la langue étant juste derrière les incisives inférieures (contact incomplet) (fig. 7).

Anormalement la pointe de la langue s'interpose entre les incisives, et parfois les bords s'interposent entre les molaires[1].

L'interposition antérieure à l'articulation du «S» est rare; elle signe une posture antérieure ou une longueur linguale importante difficile à rétracter par l'enfant[13].

\section{$>$ Troubles audibles}

Les sigmatismes sont des troubles audibles de la prononciation des consonnes, facilement décelables et caractéristiques, et vont le plus souvent orienter l'entourage du jeune enfant vers une consultation orthophonique, plutôt qu'orthodontique.

Ils sont généralement dus à l'interposition de la langue dans l'articulé dentaire entre les incisives pour le sigmatisme interdental ou zozotement $(D, T)$, ou entre les prémolaires pour le sigmatisme latéral ou schlintement $(\mathrm{S}, \mathrm{CH}, \mathrm{J})$ $[14,22,24]$.

\section{Phonation et morphogenèse}

S'il est bien établi qu'une fonction, ou dysfonction oro-faciale, contribue à la morphogenèse maxillo-faciale, tous les auteurs ne sont pas unanimes en ce qui concerne la phonation[8, 17]. La brièveté de ces appuis linguaux (1/10e de seconde) a suscité beaucoup de controverses[24] quant à l'éventuel rôle étiologique de certaines articulations phonétiques anormales dans la morphogenèse des malocclusions[8]. Ces auteurs reconnaissent toutefois à la phonation un intérêt diagnostique, mais aussi thérapeutique dans l'éducation de la langue.

La grande fréquence de ces appuis lors de l'élocution a été souvent avancée pour étayer

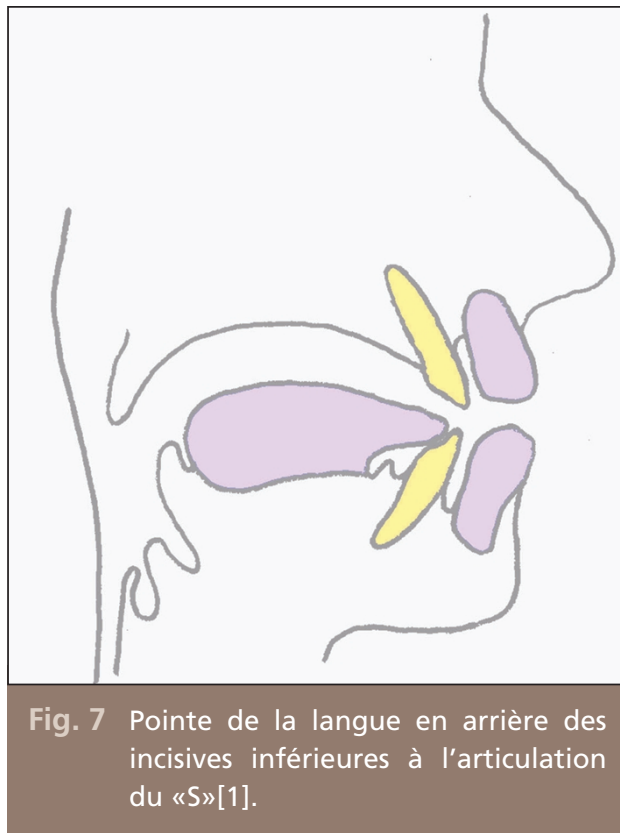

cette théorie de contribution morphogénétique[1, 22].

Fernex et Martinelli, cités par Duhart[8], ont effectué une étude sur des enfants sourdsmuets dont certains ont récupéré une «phonation quasi-normale». Il est apparu que les «parlants» et les «non-parlants» présentaient des différences morphologiques au niveau des mâchoires, qui peuvent être attribuées à des différences de fonction[8].

L'utilisation en clinique des jauges d'extensiométrie (sensibles à des pressions différentielles linguales et labiales de l'ordre de $1 \mathrm{~g}$ ) pu mettre en évidence la contribution de la phonation. Ces appareils ont permis de détecter des différences de pression observables au cours des praxies de l'articulation de la voix pouvant atteindre 20, 30 voire même $40 \mathrm{~g}$ et que la direction des procès alvéolaires était déviée dans le sens des forces musculaires dominantes[1, 22].

Trois troubles morphogénétiques sont associés à l'articulation anormale des phonèmes : 


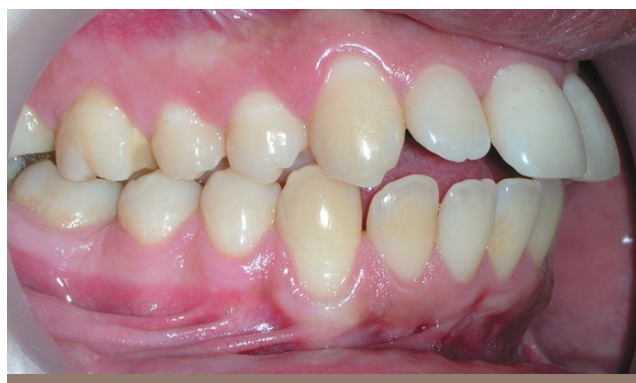

Fig. 8 Prédominance linguale : proalvéolie et béance caractéristiques.
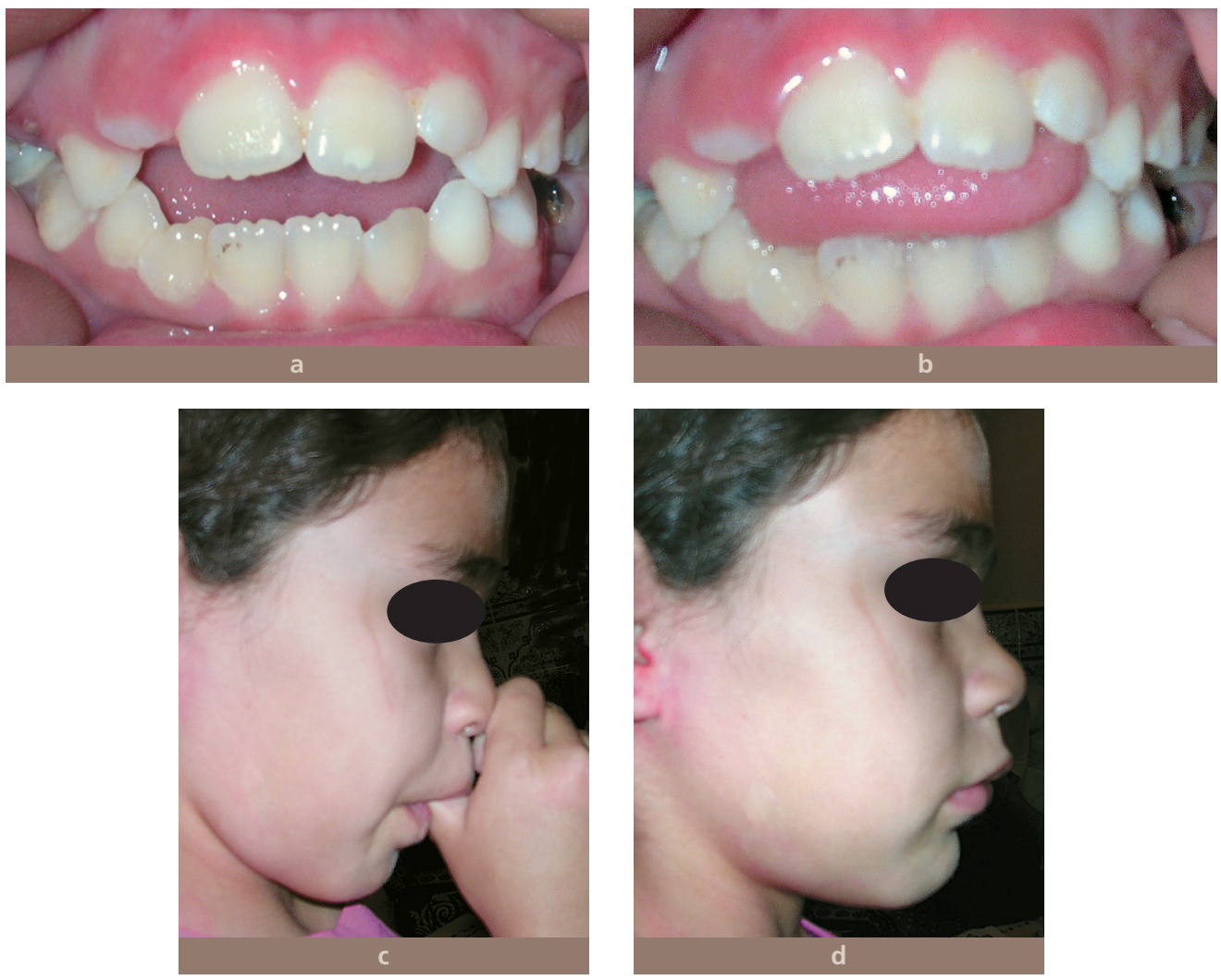

Fig. 9 a à d Béance antérieure avec interposition linguale à l'articulation du «T», associée à une succion du pouce responsable du profil de cl II squelettique.

- proalvéolie incisive supérieure: environnement musculaire déséquilibré (langue prédominante sur l'orbiculaire des lèvres) (fig. 8) ;

- béance antérieure : forces musculaires équilibrées avec interposition linguale, souvent association d'autres praxies (succion du pouce, de la lèvre inférieure...)[2] (fig. 9 a-d) ;
- supraclusie incisive : interposition latérale de la langue dans l'articulation des phonèmes $(\mathrm{S}, \mathrm{J}, \mathrm{Z}, \mathrm{Ch})$ qui retentit sur la croissance verticale des procès alvéolaires et entraîne une infra-alvéolie molaire, le plus souvent unilatérale[22] (fig. 6 a et b). 


\section{Examen clinique fonctionnel}

Lors de cet examen, on va s'intéresser beaucoup plus au contexte fonctionnel du patient :

- aspect général du patient (inhibition, passivité, agressivité, motivation et coopération) ;

- comportement familial à son égard (surprotection, indifférence) ; ces éléments laissent présager l'attitude générale face au traitement ou à la rééducation à venir ;

- observer lors de l'entretien : posture générale, position de repos des éléments faciaux, existence de tics oraux ou de parafonctions, habitudes nocives (ex : succion du pouce)[10, 13] ;

- apprécier la fonction ventilatoire (tests de Rosenthal, Gudin...) ;
- observer les mimiques de l'enfant lors de I'élocution, mais aussi lors de la déglutition (la prise de séquence vidéo permet de mieux étudier les dysfonctions éventuelles : regarder l'enfant parler pendant quelques minutes, les déglutitions sont nombreuses au cours de l'élocution; ou lui demander de boire de l'eau dans un gobelet)[10, 13, 14] ;

- la fonction linguale suscitera notre intérêt majeur : apprécier la position et la forme de la langue au repos; évaluer notamment la qualité du schéma corporel avec des tests simples de mobilité linguale "guidée» $[2,10$, 13] ; contrôler les appuis linguaux à l'articulation des linguo-palatales antérieurs notamment[13].

\section{Rééducation neuro-musculaire}

Il est clair que la phonation a un rôle morphogénétique certain durant le développement dento-facial de l'enfant; et il va de soi que la connaissance de tous ses aspects normaux et anormaux est capitale notamment pour le pédodontiste qui est souvent consulté en premier ; et qui aura le soin de convier une équipe multidisciplinaire (orthophoniste - surtout si troubles audibles associés -, orthodontiste, oto-rhino-laryngologiste si troubles ORL associés). La décision clinique va se faire en concertation avec les membres de cette équipe, et un plan de traitement global de prévention, de rééducation et de réhabilitation phonatoire peut être élaboré[27, 28].

La rééducation phonétique proprement dite, qui en est l'élément capital, est menée par l'orthophoniste.
Âge de début de la rééducation

«Attendre 6 ou 7 ans, auparavant l'enfant a encore le droit d'être petit»[11].

L'âge de 8 à 12 ans paraît raisonnable pour commencer une rééducation d'autant plus qu'il pourrait coïncider avec l'âge de l'abandon des parafonctions[1, 25].

Il sera préférable d'attendre la mise en place des incisives permanentes pour corriger une interposition linguale ou un sigmatisme addental. Quoique cette phase de transition soit la plus critique, car la chute des incisives temporaires est une occasion propice à l'interposition linguale et l'entretien d'une béance antérieure (fig. 10 a-c), et où le recours à des exercices phonétiques simples modifiant les appuis linguaux est suffisant pour assurer une 

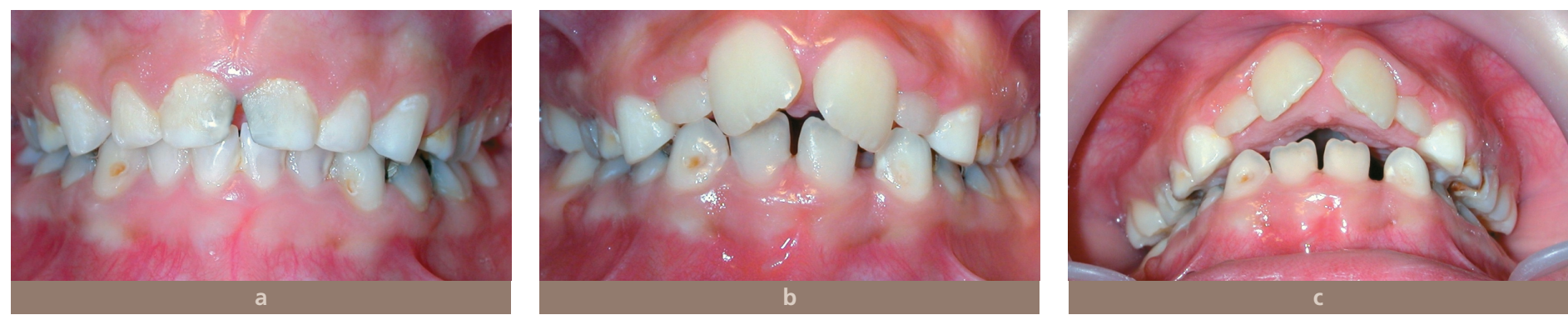

Fig. 10 a à c Dysfonction linguale et malocclusion après la chute des incisives temporaires.

transition dans les meilleures conditions (fig. 9 a et b). L'orthophoniste, en concertation avec le pédodontiste, pourrait en cas de perte prématurée des incisives temporaires indiquer une prothèse provisoire amovible, à une période où l'enfant acquiert les bons appuis phonatoires et où toute compensation erronée d'articulation n'est pas permise[23]. Cet âge correspond également à l'acquisition complète, dès 7 ans, des phonèmes par l'enfant, nécessaires à l'articulation de sa langue maternelle[1].

\section{Rééducation de la phonation}

Contrairement aux deux fonctions respiration et succion-déglutition qui sont innées, la déglutition adulte (mastication-déglutition) et la phonation sont acquises ; ce qui les rend perturbables et nécessite un apprentissage et une

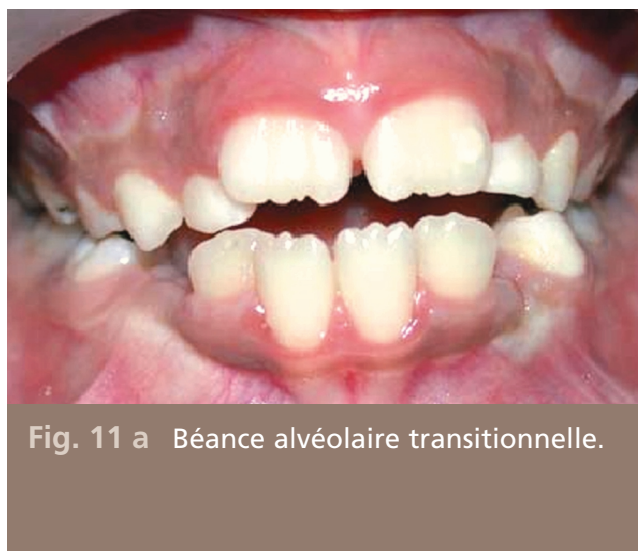

maturation à plusieurs niveaux : anatomique et fonctionnel (apparition du rempart alvéolaire et des dents), neurologique, proprioceptif (schéma corporel) et psycho-affectif[10, 16, 17, 24].

Le travail de rééducation consiste à faire prendre conscience des postures et des praxies erronées, à montrer les comportements corrects et à donner les moyens musculaires et occlusaux pour qu'elles soient aisées à acquérir et à automatiser par l'enfant $[7,11,13]$.

Elle peut être active, au moyen d'exercices phonétiques, ou passive à l'aide d'appareils adaptés prescrits par le pédodontiste ou l'orthodontiste, permettant de modifier les appuis linguaux (perle de Tucat, grille à langue (fig. 12 a-d)) ou de reposturer la langue (ELN (fig. 13), gouttière souple même en simultané à un traitement multibagues (fig. 14 a et b))[26].

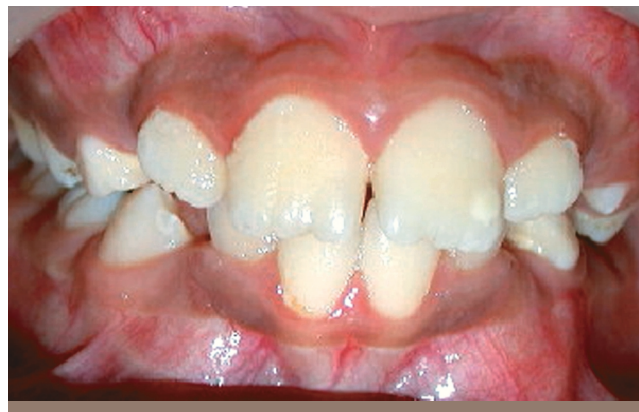

Fig. 11 b Recouvrement incisif normal après rééducation phonétique (courtoisie du Pr El Alloussi M.). 

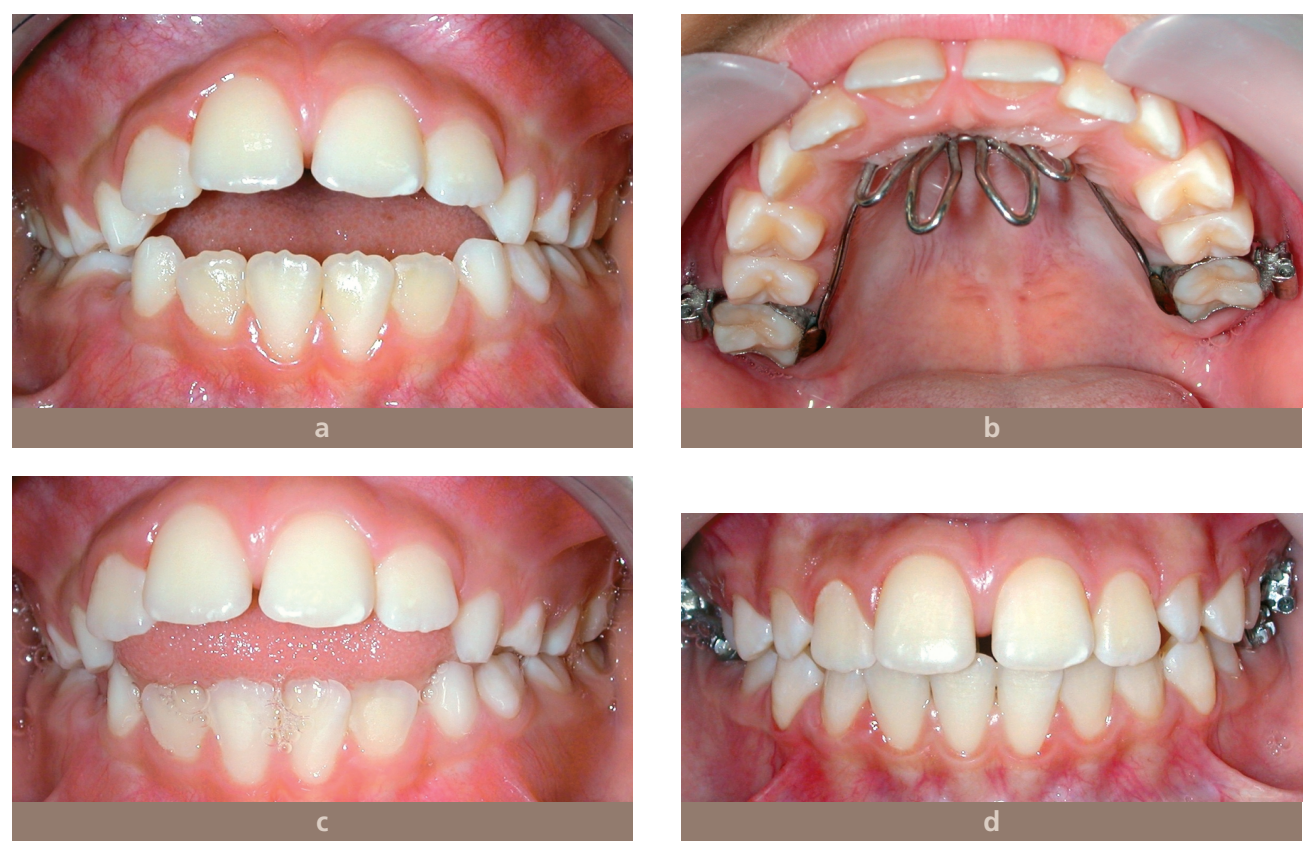

Fig. 12 a à d Correction des appuis linguaux et de la béance à l'aide d'une grille à langue.

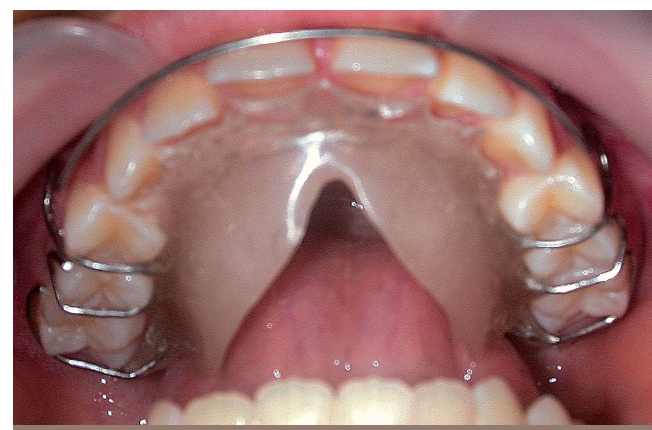

Fig. 13 Enveloppe linguale nocturne (ELN), appareil de reposturation linguale.
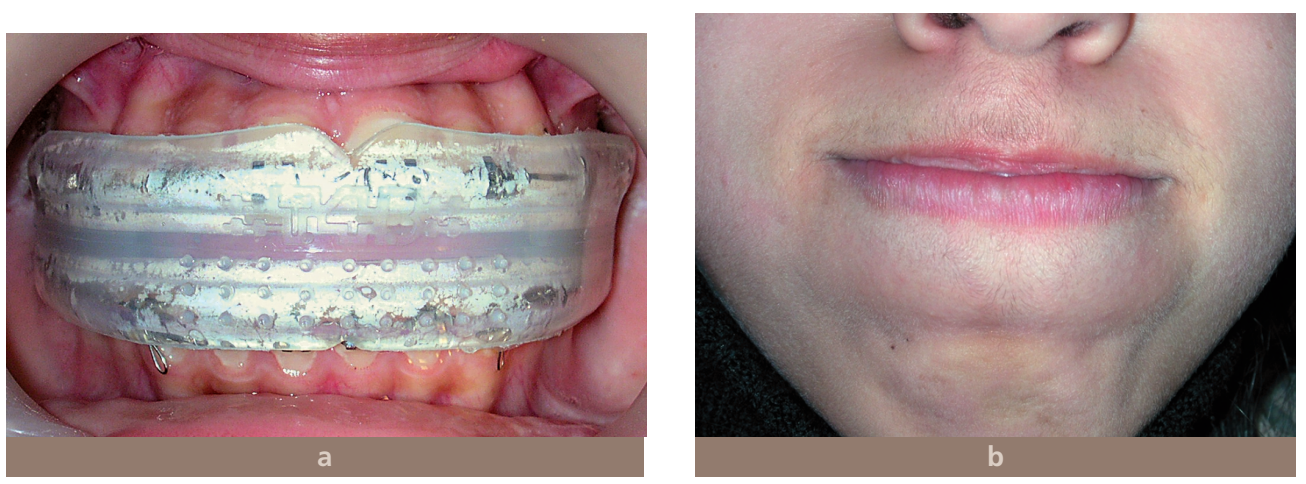

Fig. 14 a et b Gouttière souple permettant une rééducation linguale et labiale (même en simultané à un traitement orthodontique). 
Il est de règle de corriger en premier les anomalies de la déglutition qui mettent en jeu des centres cérébraux moins complexes que ceux des aires phonogènes; la rééducation phonétique est souvent négligée et même oubliée[22].

Limiter la rééducation fonctionnelle à la seule déglutition est une des raisons des échecs rencontrés.

En effet lorsque les sons sont modulés uniquement par la langue (consonnes), on peut voir durant l'élocution cette dernière s'interposer entre les arcades. Et lorsque au cours d'une phrase, le trop-plein de salive a besoin d'être éliminé, il sera plus facile à l'enfant de revenir à la succion-déglutition que d'avaler correctement (notion de confort biologique). D'où la nécessité de rééduquer les appuis linguaux lors de l'articulation des phonèmes[10, 11, 19, 26]. Lors de la première séance, on doit vérifier la position de la langue à l'émission des alvéolodentales LNDT, en faisant prononcer des mots très simples, tels que «lait», "tartine», «dînette». La plupart du temps l'articulation du L est correcte, la langue tapant très légèrement sur le palais. Cette bonne position permet de faire prendre conscience de la différence de position lors de l'émission des phonèmes NDT. Le jeune patient se rend bien compte que la langue ne se place pas sur le palais mais bien sur les dents.

Des exercices encadrés d'articulation et ensuite de tonification de la pointe de la langue vont suivre durant au moins 3 mois[11].

Les sifflantes $\mathrm{S}$ et $\mathrm{Z}$ et les chuintantes $\mathrm{CH}$ et $\mathrm{J}$, vont exiger le même soin, en insistant sur des exercices qui vont faire comprendre le mouvement vers l'arrière de la langue (mouvement inconnu de nos patients)[11].
Des séances de lecture à haute voie et à difficultés croissantes vont suivre ; le patient commence à prendre conscience de ses erreurs et les corrige donc de lui-même. Nous le faisons aussi parler car la position de la langue pendant la parole est beaucoup plus inconsciente surtout si notre patient raconte un sujet qui le passionne.

Au fur et à mesure des progrès réalisés par l'enfant, les séances deviennent plus espacées : tous les 15 jours, une fois par mois, une fois par trimestre, après les vacances scolaires. L'automatisation des bons gestes, et leur engrammation dans l'inconscient, nécessitent beaucoup de temps (minimum 3 mois) et de longues séances d'explication, de motivation et de contrôle. La réussite et la stabilité des résultats sont à ce prix[14].

\section{Rôle de l'orthodontiste dans la prise en charge du trouble d'articulé phonatoire}

Devant un trouble d'articulé phonatoire, l'orthodontiste se trouve face à deux situations :

- soit l'enfant présente peu ou pas de malocclusions associées; dans ce cas il serait judicieux d'adresser l'enfant à un orthophoniste d'autant plus s'il présente des troubles phonatoires audibles. L'orthodontiste ou le pédodontiste peuvent prescrire des appareillages type ELN, rééduquant la déglutition ou modifiant les appuis linguaux, mais ne doivent accepter aucune responsabilité quant à la correction des troubles audibles[20] ;

- soit l'enfant présente des malocclusions sévères, alvéolaires ou squelettiques, rendant difficile la découverte spontanée par l'enfant du péristaltisme lingual. 
Dans ce cas, un traitement orthodontique est nécessaire pour corriger les décalages alvéolaires et squelettiques sagittaux et transversaux avant d'avoir recours à l'orthophoniste dont le rôle est primordial pour adapter la fonction linguale au nouveau contexte occlusal, ce qui est également un gage de stabilité orthodontique[1, 9].

\section{Conclusion}

Le diagnostic et la rééducation des dysfonctions orofaciales prennent une place de plus en plus importante dans le traitement des dysmorphoses squelettiques et dento-alvéolaires. Ceci à juste titre pour pouvoir réduire les échecs et récidives des thérapeutiques basées uniquement sur le diagnostic morphologique. La phonation parmi d'autres fonctions doit faire l'objet d'un diagnostic précis et d'une rééducation neuromusculaire appropriée en cas de dysfonction.

\section{Bibliographie}

1. Ameisen E, Auclair-Assad C, Rolland ML.

Phonation et Orthodontie. Encycl Méd Chir 22-009-B-10, 2003, 10 p.

2. Bassigny $\mathrm{F}$. Examen systématique de la cavité buccale en orthodontie. Encycl Méd Chir 23-460-C-10, 1998, 12 p.

3. Bolender C, Maillard-Salin G., Simon P.

Platogramme

et palatopogramme :

méthodologie.

Orthod Fr 1970;47:549-557.

4. Bolender C, Toledano D.,

Simon P.

Platogramme,

palatopogramme :

phonation et orthodontie.

Orthod Fr 1970;47:559-574.

5. Bouvet JM.

Langage oral

et orthodontie.

Rapport du XIXe congrès de Stomatologie.

Doin, Paris, 1965.

6. Dechaume M, Cauhépé J,

Fieux J, et Noix M.

Étude

radiocinématographique

des effecteurs buccaux

(en contribution à l'étude

de la physiologie

de la déglutition

et de la phonation).

Rev Stomatol 1959;60:64-65.

7. Deneuville O.

Rééducation en orthopédie

dento-faciale,

rééducation

de la déglutition

et de la phonation.

Encycl Méd Chir 26-435-A-10, 1988, 8 p.

8. Duhart AN.

La phonation.

Orthod Fr 1992;63(1):99-102.

9. Fellus $P$.

Dysfonctions linguales

et dysmorphies.

Orthod Fr 2006;77;105-112.
10. Fellus $P$.

Orthodontie précoce

en denture temporaire.

Éditions CdP 2003.

11. Ferré JC, Fournier MY. Réadaptation fonctionnelle oro-faciale.

Encycl Méd Chir 23-495-A-10, 1996, 14 p.

12. Fieux J.

Les méthodes

filmo-radiologiques

dans l'étude des appuis

et des équilibres

de la musculature buccale.

Rev Stomatol 1961;62:1-4.

13. Fournier $M$.

La rééducation

fonctionnelle chez l'enfant et son contrôle par l'orthodontiste.

Rev Orthop Dento Faciale 1994;28:473-485.

14. François $M$.

Troubles de la voix et de l'articulation chez l'enfant. 
Encycl Méd Chir, Oto-rhino-laryngologie 2004;1:175-186.

15. Frapier L, Roos S, Hafsaoui L. Le jeu fonctionnel dans la correction et la stabilité des incisives. Int Orthod 2005;3:19-34.

16. Husson R. Physiologie de la phonation. Masson Paris, 1962.

17. Johnson NCL, Sandy JR. Tooth position and speech: is there relationship? Angle Orthod 1999;69(4):306-10.

18. Lejoyeux $\mathrm{E}$.

Les comportements oro-faciaux et leur maturation. Encycl Méd Chir 23-474-A-10, 1981, 14 p.

19. Margaillon-Fiammengo L. La phonétique : un adjuvant de choix pour la correction des tics oraux archaïques. AOS 1975;110:265-274.

20. Mason RM, Glass L. Speech and language (p. 135-149).

In Pediatric dentistry

(scientific foundations

and clinical practice)

Stewart RE, Barber TK,

Troutman KC, Wei SHY.

The C.V. Mosby Compagny,

St Louis 1982.

21. Meng $B$.

Alexandre Dumas

Orthodontiste.

Pathogénie

de la proalvéolie anglaise.

AOS 1975;109:99-101.

22. Netter JC, Bernardout $\mathrm{H}$. Phonation, odontologie et stomatologie.

Encycl Méd Chir 22-008-P-10; 1986:10 p.

23. Pinkham JR, Casamassimo PS, McTigue DJ, Fields HW, Nowak AJ.

Pediatric dentistry:

Infancy through

adolescence

(fourth edition).

Elsevier Saunders, St Louis 2005:424.

24. Romette D.

Examen des fonctions en orthopédie

dento-faciale.

Encycl Méd Chir 23-460-E-10; 1988:14 p.

25. Soulet $A$.

Rôle de la langue au cours des fonctions oro-faciales. Rev Orthop Dento Faciale 1989;23:31-52.

26. Soulet $A$.

Éducation neuro-musculaire des fonctions oro-faciales. Rev Orthop Dento Faciale 1989;23:135-175.

27. Todorova I.

Orthopédie préventive et interceptive.

Encycl Méd Chir 23-405-E-10; 1999:8p.

28. Weinberg B.

Pedodontics

and speech pathology:

Speech and language performance in children In Dentistry for the child and adolescent.

(McDonald RE, Avery DR)

Third edition.

The C.V. Mosby Compagny, St Louis 1978:515-531.

\section{Management of articulatory speech disorders}

Hicham BENYAHIA,

Loubna BAHIJE,

Fatima ZAOUI,

Elhoussine AALLOULA

$$
\begin{aligned}
& \text { Keywords } \\
& \text { - speech } \\
& \text { - articulatory speech } \\
& \text { disorders } \\
& \text { - oro-facial functions } \\
& \text { - morphogenesis } \\
& \text { - neuro-muscular } \\
& \text { rehabilitation }
\end{aligned}
$$

Functions of the oro-facial sphere, in particular ventilation and swallowing play an important part in the morphogenesis of the dental arches.

All the authors are not unanimous with regard to speech, which caused many controversies as for its morphogenetic contribution.

Recent works tend to replace this major function with its true value within the oro-facial sphere.

The articulatory speech disorders interest the orthodontist more and more, since they can be at the origin of dento-alveolar malocclusions.

The functional examination makes it possible to specify these defective lingual supports at the time of speech articulation, to direct a considered and well targeted speech rehabilitation. 


\section{Questions - Réponses}

1/ Réponse par vrai ou faux

a - La maturité intellectuelle de l'enfant est nécessaire pour la rééducation de la phonation
Vrai
Faux

b - Le contrôle des appuis linguaux lors de l'articulation des phonèmes a plus d'intérêt orthodontique, que le contrôle de la qualité du langage oral
Vrai
Faux

C - Les phonèmes linguo-palatales antérieures (T.D.N.) présentent des sites appositionnels linguaux identiques à ceux de la déglutition (au niveau de la papille rétro-incisive)

Vrai

Faux

d - La rééducation fonctionnelle de la déglutition est suffisante pour corriger les articulés linguaux lors de la phonation
Vrai
Faux

e - Le traitement orthodontique doit se faire en préalable à la rééducation orthophonique en présence de malocclusions empêchant un péristaltisme lingual correct
Vrai
Faux

2/ QCM à une seule réponse

Le traitement orthodontique doit se faire en préalable à la rééducation orthophonique en présence de :
a - malocclusions importantes
b - trouble phonatoire audible
c - malocclusions légères

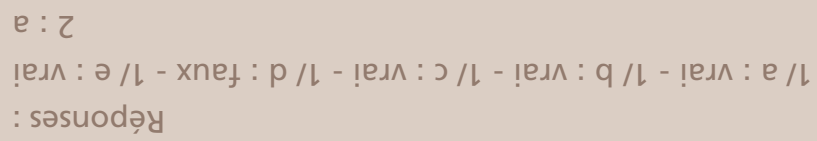

